\title{
Existence results for first derivative dependent $\phi$-Laplacian boundary value problems
}

\author{
Imran Talib and Thabet Abdeljawad ${ }^{2,3,4^{*}}$ (1)
}

"Correspondence:
tabdeljawad@psu.edu.sa
2Department of Mathematics and
General Sciences, Prince Sultan
University, Riyadh, Saudi Arabia
3Department of Medical Research,
China Medical University, 40402
Taichung, Taiwan
Full list of author information is
available at the end of the article

available at the end of the article

\begin{abstract}
Our main concern in this article is to investigate the existence of solution for the boundary-value problem

$$
\begin{aligned}
& \left(\phi\left(x^{\prime}(t)\right)^{\prime}=g_{1}\left(t, x(t), x^{\prime}(t)\right), \quad \forall t \in[0,1],\right. \\
& \Upsilon_{1}\left(x(0), x(1), x^{\prime}(0)\right)=0, \\
& \Upsilon_{2}\left(x(0), x(1), x^{\prime}(1)\right)=0,
\end{aligned}
$$

where $g_{1}:[0,1] \times \mathbb{R}^{2} \rightarrow \mathbb{R}$ is an $L^{1}$-Carathéodory function, $\Upsilon_{i}: \mathbb{R}^{3} \rightarrow \mathbb{R}$ are continuous functions, $i=1,2$, and $\phi:(-a, a) \rightarrow \mathbb{R}$ is an increasing homeomorphism such that $\phi(0)=0$, for $0<a<\infty$. We obtain the solvability results by imposing some new conditions on the boundary functions. The new conditions allow us to ensure the existence of at least one solution in the sector defined by well ordered functions. These ordered functions do not require one to check the definitions of lower and upper solutions. Moreover, the monotonicity assumptions on the arguments of boundary functions are not required in our case. An application is considered to ensure the applicability of our results.
\end{abstract}

MSC: Primary 34B15; secondary 34B24; 34C25; 34L30

Keywords: Lower and upper solutions; Coupled nonlinear system; Coupled nonlinear boundary condition; Arzelà-Ascoli theorem; Schauder's theorem

\section{Springer}

\section{Introduction}

The aim of this article is to study the existence of solution for the following nonlinear boundary value problems (BVPs) with $\phi$-Laplacian operator:

$$
\left(\phi\left(x^{\prime}(t)\right)^{\prime}=g_{1}\left(t, x(t), x^{\prime}(t)\right), \quad \forall t \in[0,1]\right.
$$

(c) The Author(s) 2020. This article is licensed under a Creative Commons Attribution 4.0 International License, which permits use, sharing, adaptation, distribution and reproduction in any medium or format, as long as you give appropriate credit to the original author(s) and the source, provide a link to the Creative Commons licence, and indicate if changes were made. The images or other third party material in this article are included in the article's Creative Commons licence, unless indicated otherwise in a credit line to the material. If material is not included in the article's Creative Commons licence and your intended use is not permitted by statutory regulation or exceeds the permitted use, you will need to obtain permission directly from the copyright holder. To view a copy of this licence, visit http://creativecommons.org/licenses/by/4.0/. 
subject to the the following generalized nonlinear boundary conditions (BCs):

$$
\left\{\begin{array}{l}
\Upsilon_{1}\left(x(0), x(1), x^{\prime}(0)\right)=0 \\
\Upsilon_{2}\left(x(0), x(1), x^{\prime}(1)\right)=0
\end{array}\right.
$$

where $g_{1}:[0,1] \times \mathbb{R}^{2} \rightarrow \mathbb{R}$ is $L^{1}$-Carathéodory function, $\Upsilon_{i}: \mathbb{R}^{3} \rightarrow \mathbb{R}(i=1,2)$ are continuous functions, and $\phi$ satisfies the hypothesis:

$\left(H_{1}\right) \phi:(-a, a) \rightarrow \mathbb{R}$ is an increasing homeomorphism such that $\phi(0)=0$, for $0<a<\infty$.

The solution of (1)-(2) is a continuously differentiable function, $x \in C^{1}[0,1]$ with $\left\|x^{\prime}\right\|_{\infty}<a$, and $\left(\phi \circ x^{\prime}\right) \in \mathrm{AC}[0,1]$, which satisfies (1) and (2).

The BVPs (1) with $x^{\prime \prime}$ instead of $\left(\phi\left(x^{\prime}(t)\right)^{\prime}\right.$ has been studied extensively in the literature employing a lower and upper solutions approach, a topological approach, degree and fixed point index theory, and fixed point and continuation theorems; see for example [1-11], and the references therein. These approaches usually make use of an appropriate Green function, which, for the linear operator $x \mapsto x_{1}^{\prime \prime}$, can be found and successfully handled. In recent times, the researchers put their efforts to developing the existence and localization results for the BVPs with a $\phi$-Laplacian operator, $x \mapsto\left(\phi\left(x^{\prime}(t)\right)^{\prime}\right.$. Such types of BVPs are solved with classical BCs, like periodic, anti-periodic, Dirichlet, Neumann, and mixed cases; see for example [12-27], and the references therein.

The study of $\phi$-Laplacian equations is a classical topic having applications in glaciology, population biology, nonlinear flow laws, non-Newtonian mechanics, and combustion theory; see for example [28-31], and the references therein. Moreover, the $\phi$-Laplacian operators are involved in some models, e.g., in non-Newtonian fluid theory, diffusion of flows in porous media, nonlinear elasticity, and theory of capillary surfaces [32].

A lower and upper solutions (LUSs) approach is widely investigated to develop the existence and solvability results for classical and fractional order differential equations with $\phi$-Laplacian operator; see for example [33-37] and the references therein.

Motivated by the above-mentioned work on $\phi$-Laplacian differential equations, we develop an existence criterion for the boundary value problem (BVP), (1)-(2). To the best of our knowledge, (1) is still an untreated problem with BCs (2). The approach we use in our study is simpler than the approaches used in $[1,3,33,34]$. We prove the existence of solutions of (1)-(2) by using new conditions given on the BCs (2). These conditions allow us to obtain a solution in the sector defined by well-ordered functions. These ordered functions do not require one to check the definitions of LUSs. Moreover, the requirement to impose the monotonicity assumptions on the arguments of the $\mathrm{BCs}$ is not necessary in our case. The arguments we use in our study are the Arzelà-Ascoli and Schauder's fixed point theorems.

The rest of the article is organized as follows: in Sect. 2, we present preliminary definitions and auxiliary results, in Sect. 3, we prove the existence of solutions of the problem (1)-(2), in Sect. 4, we consider an example to verify the results of Sect. 3, and in Sect. 5, the conclusion is given.

\section{Preliminaries}

The following definitions are helpful in understanding the main result.

Definition 1 Let $x \in C^{1}([0,1])$ be a solution of (1)-(2), if $\left\|x^{\prime}\right\|_{\infty}<a,\left(\phi \circ x^{\prime}\right) \in \mathrm{AC}[0,1]$, and satisfy (1)-(2). 
Definition 2 The function $g_{1}:[0,1] \times \mathbb{R}^{2} \rightarrow \mathbb{R}$ is $L^{1}$-Carathéodory if:

1. $g_{1}(\cdot, x, y):[0,1] \rightarrow \mathbb{R}$ is measurable for all $(x, y) \in \mathbb{R}^{2}$,

2. $g_{1}(t, ., .):, \mathbb{R}^{2} \rightarrow \mathbb{R}$ is continuous for a.e. $t \in[0,1]$,

3. for each compact set $A \subset \mathbb{R}^{2}$, there is a function $\mu_{A} \in L^{1}$, such that

$$
\left|g_{1}(t, x, y)\right| \leq \mu_{A}(t)
$$

for a.e. $t \in[0,1]$ and all $(x, y) \in A$.

The following lemmas are very useful for obtaining our main results.

Lemma 2.1 For each $(k, g) \in C([0,1]) \times(-a, a)$, we have a unique $d:=\mathbf{D}_{\phi}(k, g)$, such that

$$
\int_{0}^{1} \phi^{-1}(k(t)-d) d t=g
$$

Also, $\mathbf{D}_{\phi}: C([0,1]) \times(-a, a) \longrightarrow \mathbb{R}$ is a continuous function.

Proof First, the uniqueness and existence is shown, then continuity. Let, for $(k, g) \in$ $C([0,1]) \times(-a, a)$, there exist $d_{1}, d_{2}$ such that

$$
\int_{0}^{1} \phi^{-1}\left(k(t)-d_{1}\right) d t=g=\int_{0}^{1} \phi^{-1}\left(k(t)-d_{2}\right) d t .
$$

For some $s \in[0,1]$, we have

$$
\int_{0}^{1} \phi^{-1}\left(k(s)-d_{1}\right) d t=g=\int_{0}^{1} \phi^{-1}\left(k(s)-d_{2}\right) d t
$$

Since $\phi^{-1}$ is injective function, (5) implies that

$$
\phi^{-1}\left(k(s)-d_{1}\right)=\phi^{-1}\left(k(s)-d_{2}\right) .
$$

Equation (6) further implies

$$
k(s)-d_{1}=k(s)-d_{2},
$$

hence, $d_{1}=d_{2}$. Now define a function, $\delta: \mathbb{R} \rightarrow \mathbb{R}$, such that

$$
\delta(s)=\int_{0}^{1} \phi^{-1}(k(t)-s) d t
$$

is well defined, decreasing, and continuous. Also

$$
\lim _{s \rightarrow \infty} \delta(s)=a, \quad \lim _{s \rightarrow-\infty} \delta(s)=-a .
$$

Equation (9) implies the existence of a unique $d=\mathbf{D}_{\phi}(k, g)$. Now, it remains to show that $\mathbf{D}_{\phi}: C([0,1]) \times(-a, a) \longrightarrow \mathbb{R}$ is a continuous function. 
Let $\left(k_{n}, g_{n}\right) \subset C([0,1]) \times(-a, a)$ and $\left(k_{n}, g_{n}\right) \longrightarrow\left(k_{0}, g_{0}\right)$ in $C([0,1]) \times(-a, a)$. Now let $\mathbf{D}_{\phi}\left(k_{n}, g_{n}\right)$ be a subsequence converges to $d_{0}$, then by the application of the dominated convergence theorem, we deduce that $\int_{0}^{1} \phi^{-1}\left(k_{0}(t)-d_{0}\right) d t=g_{0}$, which implies $\mathbf{D}_{\phi}\left(k_{0}, g_{0}\right)=$ $d_{0}$. Hence, $\mathbf{D}_{\phi}: C([0,1]) \times(-a, a) \longrightarrow \mathbb{R}$ is a continuous function.

\section{Main results}

Theorem 1 Suppose we have the existence of ordered functions $\eta_{1}, \eta_{2} \in C[0,1]$, such that, $\eta_{1}(0)<\eta_{2}(0)$ and $\eta_{1}(1)<\eta_{2}(1)$,

$$
\begin{aligned}
& \max \left\{\left|\eta_{2}(1)-\eta_{1}(0)\right| ;\left|\eta_{1}(1)-\eta_{2}(0)\right|\right\}<a, \\
& \Upsilon_{1}\left(\eta_{1}(0), t, w\right) \geq 0, \quad \forall(t, w) \in\left[\eta_{1}(0), \eta_{2}(0)\right] \times[-a, a], \\
& \Upsilon_{1}\left(\eta_{2}(0), t, w\right) \leq 0, \quad \forall(t, w) \in\left[\eta_{1}(0), \eta_{2}(0)\right] \times[-a, a], \\
& \eta_{1}(1)-\Upsilon_{2}\left(x, \eta_{1}(1), z\right) \leq 0, \quad \forall x, z \in\left[\eta_{1}(0), \eta_{2}(0)\right], \\
& \eta_{2}(1)-\Upsilon_{2}\left(x, \eta_{2}(1), z\right) \geq 0, \quad \forall x, z \in\left[\eta_{1}(0), \eta_{2}(0)\right] .
\end{aligned}
$$

Then the problem (1)-(2) has at least one solution, such that $\eta_{1}(0)-a t \leq x(t) \leq \eta_{2}(0)+a t$, for all $t \in[0,1]$.

Proof Suppose we have the following modified BVP:

$$
\left(\phi\left(x^{\prime}(t)\right)^{\prime}=G(x)(t), \quad \text { a.e. } t \in[0,1]\right.
$$

with modified BCs

$$
\begin{aligned}
& x(0)=\mathbf{A}(x), \\
& x(1)=\mathbf{B}(x),
\end{aligned}
$$

where

$$
G(x)(t)=g_{1}\left(t, \rho(t, x(t)), \varrho\left(x^{\prime}(t)\right)\right)
$$

with

$$
\begin{aligned}
& \rho(t, x(t))=\max \left\{\eta_{1}(0)-a t, \min \left\{x, \eta_{2}(0)+a t\right\}\right\}, \\
& \varrho\left(x^{\prime}(t)\right)=\max \{-a, \min \{x, a\}\},
\end{aligned}
$$

and

$$
\begin{aligned}
& \mathbf{A}(x)=\sigma\left(x(0)+\Upsilon_{1}\left(x(0), x(1), x^{\prime}(0)\right)\right), \\
& \mathbf{B}(x)=\chi\left(\frac{1}{2} x(1)+\frac{1}{2} \Upsilon_{2}\left(x(0), x(1), x^{\prime}(1)\right)\right),
\end{aligned}
$$

with

$$
\sigma(x)=\max \left\{\eta_{1}(0), \min \left\{x, \eta_{2}(0)\right\}\right\},
$$




$$
\chi(x)=\max \left\{\eta_{1}(1), \min \left\{x, \eta_{2}(1)\right\}\right\} .
$$

For simplicity, we divide the proof in three steps.

Step 1: The solution of the problem (11)-(12) is equivalent to find a fixed points of the operator, $\Omega: C^{1}[0,1] \longrightarrow C^{1}[0,1]$ defined as

$$
\Omega(x)(t)=\mathbf{A}(x)+\int_{0}^{t} \phi^{-1}\left[\lambda_{x}+\int_{0}^{s} G(x)(x) d x\right] d s, \quad \forall t \in[0,1] .
$$

Firstly, we ensure the existence of $\lambda_{x} \in \mathbb{R}$. For this, we claim that for each $x \in C^{1}[0,1]$, there exists a unique $\lambda_{x} \in \mathbb{R}$, such that

$$
\int_{0}^{1}\left(\phi^{-1}\left[\lambda_{x}+\int_{0}^{t} G(x)(s) d s\right]\right) d t=\mathbf{B}(x)-\mathbf{A}(x) .
$$

Let

$$
k(t)=\int_{0}^{t} G(x)(s) d s \in C[0,1]
$$

and

$$
g=\mathbf{B}(x)-\mathbf{A}(x)
$$

Obviously,

$$
|g| \leq|\mathbf{B}(u)-\mathbf{A}(u)| \leq \max |\beta(1)-\alpha(0) ; \alpha(1)-\beta(0)|<a .
$$

Consequently, $\lambda_{x} \in \mathbb{R}$ exists by Lemma 2.1 .

Since $G(x)$ is bounded and continuous on $[0,1]$, and the integral is a continuous function on $[0, s]$. Furthermore, $\lambda_{x}$ exists, $\phi$ is a homomorphism and its inverse exists. Also, $\phi^{-1}\left[\lambda_{x}+\int_{0}^{s} G(x)(x) d x\right]$ is continuous, and its integral exists. Therefore $\Omega(x)$ is continuous on $[0,1]$. Further, the class $\left\{\Omega(x): x \in C^{1}[0,1]\right\}$ is uniformly bounded and equicontinuous. Therefore in view of the Arzelà-Ascoli theorem $\left\{\Omega(x): x \in C^{1}[0,1]\right\}$ is relatively compact. Consequently $\Omega$ is a compact map. Now the Schauder fixed point theorem guarantees the existence of at least a fixed point since $\Omega$ is continuous and compact.

Step 2: If $x(t), t \in[0,1]$ be a solution of the problem (11)-(12), then it must satisfy

$$
\eta_{1}(0)-a t<x(t)<\eta_{2}(0)+a t, \quad \forall t \in[0,1] .
$$

Since $x(0)=\mathbf{A}(x)$,

$$
\begin{aligned}
x(0) & =\sigma\left(x(0)+\Upsilon_{1}\left(x(0), x(1), x^{\prime}(0)\right)\right) \\
& =\max \left\{\eta_{1}(0), \min \left\{x(0)+\Upsilon_{1}\left(x(0), x(1), x^{\prime}(0)\right), \eta_{2}(0)\right\}\right\} \\
& =\eta_{2}(0) .
\end{aligned}
$$

Now, we show that

$$
\eta_{1}(0) \leq x(0)+\Upsilon_{1}\left(x(0), x(1), x^{\prime}(0)\right) \leq \eta_{2}(0) .
$$


Suppose on the contrary that

$$
\begin{aligned}
& \eta_{2}(0)<x(0)+\Upsilon_{1}\left(x(0), x(1), x^{\prime}(0)\right), \\
& \text { then } \\
& \eta_{2}(0)-x(0)<\Upsilon_{1}\left(x(0), x(1), x^{\prime}(0)\right), \\
& 0<\Upsilon_{1}\left(\eta_{2}(0), x(1), x^{\prime}(0)\right),
\end{aligned}
$$

which implies a contradiction, because $\Upsilon_{1}\left(\eta_{2}(0), x(1), x^{\prime}(0)\right) \ngtr 0$. Hence

$$
x(0)+\Upsilon_{1}\left(x(0), x(1), x^{\prime}(0)\right) \leq \eta_{2}(0) .
$$

Along the same lines, we can show that

$$
\eta_{1}(0) \leq x(0)+\Upsilon_{1}\left(x(0), x(1), x^{\prime}(0)\right) .
$$

Using (21) and (22), we have

$$
\eta_{1}(0) \leq x(0) \leq \eta_{2}(0)
$$

Now using the second boundary condition, $x(1)=\mathbf{B}$, we may show that

$$
\eta_{1}(1) \leq x(1) \leq \eta_{2}(1)
$$

Since $\left\|x^{\prime}\right\|_{\infty}<a$,

$$
x(0)-a t<x(t)<x(0)+a t, \quad \forall t \in[0,1] .
$$

Using Eqs. (23)-(25), we have

$$
\eta_{1}(0)-a t<x(t)<\eta_{2}(0)+a t, \quad \forall t \in[0,1] .
$$

Step3: If $x(t)$ is a solution of the problem (11)-(12), then it must satisfy the BCs

$$
\left\{\begin{array}{l}
\Upsilon_{1}\left(x(0), x(1), x^{\prime}(0)\right)=0, \\
\Upsilon_{2}\left(x(0), x(1), x^{\prime}(1)\right)=0
\end{array}\right.
$$

To satisfy the BCs (27), it is sufficient to show that

$$
\left\{\begin{array}{l}
\eta_{1}(0) \leq x(0)+\Upsilon_{1}\left(x(0), x(1), x^{\prime}(0)\right) \leq \eta_{2}(0), \\
\eta_{1}(1) \leq \frac{1}{2} x(1)+\frac{1}{2} \Upsilon_{2}\left(x(0), x(1), x^{\prime}(1)\right) \leq \eta_{2}(1) .
\end{array}\right.
$$

Suppose on the contrary that

$$
\eta_{1}(0) \geq x(0)+\Upsilon_{1}\left(x(0), x(1), x^{\prime}(0)\right) .
$$


Then

$$
\begin{aligned}
x(0) & =\sigma\left(x(0)+\Upsilon_{1}\left(x(0), x(1), x^{\prime}(0)\right)\right) \\
& =\max \left\{\eta_{1}(0), \min \left\{x(0)+\Upsilon_{1}\left(x(0), x(1), x^{\prime}(0)\right), \eta_{2}(0)\right\}\right\} \\
& =\alpha(0) .
\end{aligned}
$$

Using (29) and (30), we have

$$
\Upsilon_{1}\left(\eta_{1}(0), x(1), x^{\prime}(0)\right)<0 .
$$

This is a contradiction, because $\Upsilon_{1}\left(\eta_{1}(0), x(1), x^{\prime}(0)\right) \nless 0$. Hence

$$
\eta_{1}(0) \leq x(0)+\Upsilon_{1}\left(x(0), x(1), x^{\prime}(0)\right) .
$$

Similarly, we can show that

$$
\eta_{2}(0) \geq x(0)+\Upsilon_{1}\left(x(0), x(1), x^{\prime}(0)\right) .
$$

Now, assume on contrary that

$$
\eta_{2}(1)<\frac{1}{2} x(1)+\frac{1}{2} \Upsilon_{2}\left(x(0), x(1), x^{\prime}(1)\right) .
$$

Then

$$
\begin{aligned}
x(1) & =\mathbf{B}(x) \\
& =\chi\left(\frac{1}{2} x(1)+\frac{1}{2} \Upsilon_{2}\left(x(0), x(1), x^{\prime}(1)\right)\right) \\
& =\max \left\{x(1), \min \left\{\frac{1}{2} x(1)+\frac{1}{2} \Upsilon_{2}\left(x(0), x(1), x^{\prime}(1)\right), \eta_{2}(1)\right\}\right\} \\
& =\eta_{2}(1) .
\end{aligned}
$$

Using Eqs. (34) and (35), we have

$$
\eta_{2}(1)-\Upsilon_{2}\left(x(0), \eta_{2}(1), x^{\prime}(1)\right)<0,
$$

which implies a contradiction, because $\eta_{2}(1)-\Upsilon_{2}\left(x(0), \eta_{2}(1), x^{\prime}(1)\right) \nless 0$. Hence

$$
\eta_{2}(1) \geq \frac{1}{2} x(1)+\frac{1}{2} \Upsilon_{2}\left(x(0), x(1), x^{\prime}(1)\right) .
$$

Along the same line, we can show that

$$
\eta_{1}(1) \leq \frac{1}{2} x(1)+\frac{1}{2} \Upsilon_{2}\left(x(0), x(1), x^{\prime}(1)\right) .
$$

Using Eqs. (32), (33), (37), and (38), the BCs (27) are satisfied.

Hence, $x(t), t \in[0,1]$ is a solution of the modified BVP (11)-(12) which leads to the solution of the BVP (1)-(2). 


\section{Example}

Consider the following nonlinear BVP:

$$
\left(\frac{x^{\prime}(t)}{1-\left|x^{\prime}(t)\right|}\right)^{\prime}=x^{3}(t)+\frac{x(t)}{\sqrt{t}}+\sin \left(x^{\prime}(t)\right), \quad \text { for a.e. } t \in[0,1],
$$

subject to following nonlinear BCs:

$$
\begin{aligned}
& \Upsilon_{1}\left(x(0), x(1), x^{\prime}(0)\right)=x(0) x(1)\left(x^{\prime}(0)-3\right)=0, \\
& \Upsilon_{2}\left(x(0), x(1), x^{\prime}(1)\right)=x(0) x(1)\left(x^{\prime}(1)-5\right)=0 .
\end{aligned}
$$

This problem is a particular case of the problem (1)-(2) with

$$
\begin{aligned}
& g_{1}(t, x, y)=x^{3}(t)+\frac{x(t)}{\sqrt{t}}+\sin \left(y^{\prime}(t)\right), \quad t \in[0,1] \\
& \phi(x)=\frac{x}{\sqrt{1-|x|}}, \\
& \text { and } \\
& a=1 .
\end{aligned}
$$

\section{Choose}

$$
\eta_{1}(t)=0, \eta_{2}(t)=\frac{1}{2}, \quad \forall t \in[0,1]
$$

with

$$
\eta_{1}(0)<\eta_{2}(0), \quad \eta_{1}(1)<\eta_{2}(1)
$$

Now

$$
\begin{aligned}
& \max \left\{\left|\eta_{2}(1)-\eta_{1}(0)\right|,\left|\eta_{1}(1)-\eta_{2}(0)\right|\right\}=\frac{1}{2}<1=a, \\
& \Upsilon_{1}\left(\eta_{1}(0), t, w\right)=\eta_{1}(0) t(w-3)=0, \quad \forall(t, w) \in\left[0, \frac{1}{2}\right] \times[-1,1], \\
& \Upsilon_{1}\left(\eta_{2}(0), t, w\right)=\eta_{2}(0) t(w-3) \leq 0, \quad \forall(t, w) \in\left[0, \frac{1}{2}\right] \times[-1,1], \\
& \eta_{1}(1)-\Upsilon_{2}\left(x, \eta_{1}(1), z\right)=\eta_{1}(1)-x \eta_{1}(1)(z-5)=0, \quad \forall(x, z) \in\left[0, \frac{1}{2}\right], \\
& \eta_{2}(1)-\Upsilon_{2}\left(x, \eta_{2}(1), z\right)=\eta_{2}(1)-x \eta_{2}(1)(z-5) \geq 0, \quad \forall(x, z) \in\left[0, \frac{1}{2}\right] .
\end{aligned}
$$

Hence by Theorem 1, the BVP (39)-(40) has at least one solution, satisfying

$$
\eta_{1}(0)-a t \leq x(t) \leq \eta_{2}(0)+a t, \quad \forall t \in[0,1] .
$$




\section{Conclusion}

We studied the existence of solutions of $\phi$-Laplacian boundary value problems by employing the topological approach and the new conditions on the boundary functions. The conditions on the boundary functions allowed us to obtain a solution in the sector defined by two well-ordered functions which did not require satisfying the differential inequalities to ensure the existence of lower and upper solutions. This way to deal with the $\phi$-Laplacian boundary value problems made our approach simpler than the lower and upper solutions approach. Moreover, in our approach the monotonicity assumptions on the arguments of the boundary functions are not required. We considered an example to check the applicability of the developed theoretical results.

\section{Acknowledgements}

The author Thabet Abdeljawad would like to thank Prince Sultan University for funding this work through research group Nonlinear Analysis Methods in Applied Mathematics (NAMAM) group number RG-DES-2017-01-17.

\section{Funding}

Not applicable.

Availability of data and materials

Not applicable.

\section{Competing interests}

The authors declare that they have no competing interests.

Authors' contributions

All authors contributed equally to this article. All authors read and approved the final manuscript.

\section{Author details}

1 Department of Mathematics, Faculty of Science and Technology, Virtual University of Pakistan, 54-Lawrence Road, Lahore, Pakistan. '2Department of Mathematics and General Sciences, Prince Sultan University, Riyadh, Saudi Arabia. ${ }^{3}$ Department of Medical Research, China Medical University, 40402 Taichung, Taiwan. ${ }^{4}$ Department of Computer Science and Information Engineering, Asia University, Taichung, Taiwan.

\section{Publisher's Note}

Springer Nature remains neutral with regard to jurisdictional claims in published maps and institutional affiliations.

Received: 12 July 2020 Accepted: 24 September 2020 Published online: 01 October 2020

\section{References}

1. Talib, I., Asif, N.A., Tunc, C.: Coupled lower and upper solution approach for the existence of solutions of nonlinear coupled system with nonlinear coupled boundary conditions. Proyecciones 35, 97-115 (2016)

2. Talib, I., Asif, N.A., Tunc, C.: Existence of solutions to second-order nonlinear coupled systems with nonlinear coupled boundary conditions. Electron. J. Differ. Equ. 313, 1 (2015)

3. Asif, N.A., Talib, I., Tunc, C.: Existence of solution for first-order coupled system with nonlinear coupled boundary conditions. Bound. Value Probl. 2015, 134, 1-9 (2015)

4. Bai, Z., Du, Z.: Positive solutions for some second-order four-point boundary value problems. J. Math. Anal. Appl. 330, 34-50 (2007)

5. Bai, Z., Li, W., Ge, W.: Existence and multiplicity of solutions for four-point boundary value problems at resonance. Nonlinear Anal. 60, 1151-1162 (2005)

6. Kwong, M.K., Wong, J.S.W.: An optimal existence theorem for positive solutions of a four-point boundary value problem. Electron. J. Differ. Equ. 165, 1 (2009)

7. Schmitt, K.: On the existence of nontrivial solutions of differential equations subject to linear constraints

8. Webb, J.R.L.: Existence of positive solutions for a thermostat model. Nonlinear Anal., Real World Appl. 13, 923-938 (2012)

9. Infante, G., Pietramala, P.: Nonzero radial solutions for a class of elliptic systems with nonlocal BCs on annular domains. Nonlinear Differ. Equ. Appl. 22, 979-1003 (2015)

10. Tisdell, C.C.: Existence of solutions to second-order boundary value problems without growth restrictions. Electron. J. Qual. Theory Differ. Equ. 92, 1 (2016)

11. Infante, G., Webb, J.R.L.: Loss of positivity in a nonlinear scalar heat equation. Nonlinear Differ. Equ. Appl. 13, 249-261 (2006)

12. Bai, Z., Zhang, Y.: Solvability of fractional three-point boundary value problems with nonlinear growth. Appl. Math Comput. 218, 1719-1725 (2011)

13. Bereanu, C., Jebelean, P.: Radial solutions for some nonlinear problems involving mean curvature operators in Euclidean and Minkowski spaces. Proc. Am. Math. Soc. 137, 161-169 (2009) 
14. Bereanu, C., Jebelean, P., Mawhin, J.: Radial solutions for Neumann problems involving mean curvature operators in Euclidean and Minkowski spaces. Math. Nachr. 283, 379-391 (2010)

15. Bereanu, C., Jebelean, P., Mawhin, J.: Periodic solutions of pendulum-like perturbations of singular and bounded ö-Laplacians. J. Dyn. Differ. Equ. 22, 463-471 (2010)

16. Brezis, H., Mawhin, J: Periodic solutions of the forced relativistic pendulum. Differ. Integral Equ. 23, 801-810 (2010)

17. Coelho, I., Corsato, C., Obersnel, F., Omari, P.: Positive solutions of the Dirichlet problem for the one-dimensional Minkowski-curvature equation. Adv. Nonlinear Stud. 12, 621-638 (2012)

18. Feng, M., Li, P., Sun, S.: Symmetric positive solutions for fourth-order n-dimensional m-Laplace system. Bound. Value Probl. 2018, 63 (2018)

19. Herlea, D.R., Precup, R.: Existence, localization and multiplicity of positive solutions to $\phi$-Laplace equations and systems. Taiwan. J. Math. 20, 77-89 (2016)

20. Jebelean, P., Mahwin, J., Cerban, C.: A vector p-Laplacian type approach to multiple periodic solutions for the p-relativistic operator. Commun. Contemp. Math. 19, 1-16 (2017)

21. Jebelean, P., Precup, R.: Symmetric positive solutions to a singular $\phi$-Laplace equation. J. Lond. Math. Soc. 99, 495-515 (2019)

22. Jebelean, P., Cerban, C.: Boundary value problems for discontinuous perturbations of singular $\phi$-Laplacian operator. J. Math. Anal. Appl. 431, 662-681 (2015)

23. Precup, R., Rodríguez-López, J.: Positive solutions for discontinuous problems with applications to $\phi$-Laplacian equations. J. Fixed Point Theory Appl. 2018, 156, 1-17 (2018)

24. Sheng, K., Zhang, W., Bai, Z.: Positive solutions to fractional boundary value problems with $\mathrm{p}$-Laplacian on time scales. Bound. Value Probl. 2018, 70, 1-17 (2018)

25. Herlea, Y.R.: Existence, localization and multiplicity of positive solutions for the Dirichlet BVP with $\phi$-Laplacian. Fixed Point Theory 18, 237-246 (2017)

26. Chinní, A., Bella, B.D., Jebelean, P., Precup, R.: A four-point boundary value problem with singular $\phi$-Laplacian. J. Fixed Point Theory Appl. 21, 66 (2019)

27. Guefaifia, R., Boulaaras, S., Kamache, F.: On the existence of three solutions of Dirichlet fractional systems involving the p-Laplacian with Lipschitz nonlinearity. Bound. Value Probl. 2020, 131 (2020)

28. Agarwal, R.P., Regan, D.O., Stanek, S.: General existence principles for nonlocal boundary value problems with $\phi$-Laplacian and their applications. Abstr. Appl. Anal. 2006, 30 (2006)

29. Glowinski, R., Rappaz, J.: Approximation of a nonlinear elliptic problem arising in a non-Newtonian fluid flow model in glaciology. JMathematical Modelling and Numerical Analysis 37, 175-186 (2003)

30. Chen, T., Liu, W.: An anti-periodic boundary value problem for the fractional differential equation with a $p$-Laplacian operator. Appl. Math. Lett. 25, 1671-1675 (2012)

31. Diaz, J.I., Thélin, F.D.: On a nonlinear parabolic problem arising in some models related to turbulent flows. SIAM J. Math. Anal. 25, 1085-1111 (1994)

32. Calamai, A., Marcelli, C., Papalini, F.: Boundary value problems for singular second order equations. Fixed Point Theory Appl. 2018, 20 (2018)

33. Behi, D.A., Adje, A., Goli, K.C.: Lower and upper solutions method for nonlinear second-order differential equations involving a $\phi$-Laplacian operator. Afr. Diaspora J. Math. 22, 22-41 (2019)

34. Jiang, D.: Upper and lower solution method and a singular boundary value problem for the one-dimensional p-Laplacian. J. Math. Anal. Appl. 22, 22-41 (2019)

35. Liu, X., Jia, M.: The method of lower and upper solutions for the general boundary value problems of fractional differential equations with $p$-Laplacian. Adv. Differ. Equ. 2018, 28, 1-15 (2018)

36. Wang, J., Xiang, H.: Upper and lower solutions method for a class of singular fractional boundary value problems with p-Laplacian operator. Abstr. Appl. Anal. 2010, 1 (2010)

37. Guo, Y., Ge, W.: Upper and lower solution method and a singular boundary value problem for the one-dimensional p-Laplacian. J. Math. Anal. Appl. 252, 631-648 (2000)

\section{Submit your manuscript to a SpringerOpen ${ }^{\circ}$ journal and benefit from:}

- Convenient online submission

- Rigorous peer review

- Open access: articles freely available online

- High visibility within the field

Retaining the copyright to your article

Submit your next manuscript at $\gg$ springeropen.com 